\title{
Isolation and Enumeration of Escherichia coli from Soil and Water
}

\author{
Anindita Bhowmik ${ }^{1}$ and Sunjukta Ahsan ${ }^{1^{*}}$ \\ Department of Microbiology, University of Dhaka, Dhaka 1000, Bangladesh
}

\begin{abstract}
Majority of the population of Bangladesh depend on tap or surface water as their source of water supply. This study was carried out to examine the microbial quality of both water and soil collected from different places using the multiple tube fermentation technique to determine coliform count by the most probable number (MPN) method in brilliant green lactose broth (BGLB) media.Inoculum from positive tubes of the presumptive test were further transferred on eosinemethylene blue (EMB) and MacConkey agar.The organisms isolated were further characterized using biochemical tests. Out of 93 water samples, $30(32.26 \%)$ indicated the presence of lactose fermenter and gas producer in all 3 tubes of dilution series using inoculum quantities of 1.0, 0.1 and $0.01 \mathrm{ml}$, whereas out of 85 soil samples, 45 (52.94\%) showed acid and gas production in all 3 tubes of dilution series.Among 85 soil samples, 40 samples that contained at least one positive in each dilution series and among 93 water samples, 31 samples that contained at least one positive in each dilution series were further re-identified with biochemical tests.This study showed $\mathbf{3 0 . 5 9} \%$ soil isolates and $\mathbf{2 6 . 8 8} \%$ water isolates were Escherichia coli which highlighted the fact that both water and soil act as a major reservoir of E.coli, which indicates possible fecal contamination as well as presence of potentially pathogenic $E$. coli.
\end{abstract}

Key words:Escherichia coli, Fecal contamination.

\section{Introduction}

Water is one of the basic componentsfor man's continued existence ${ }^{1}$. Water plays a central role in the regulation of nutrient transport, toxic waste removal, thermal regulation and digestion and digestion, organ functioning and metabolic activities. But fecally contaminated water is able to spread diseases in consumers to a great number of people ${ }^{2}$.The World Health Organization estimated in 2000 that there are four billion cases of diarrhea each year in addition to millions to other casesof illness associated with the lack access of clean water ${ }^{3}$. It is recommended that water supplies contaminated with human and animal excreta particularly feces can transmit infectious diseases ${ }^{4}$. Out breaks of water borne diseases continue to occur throughout the world but are especially serious in developing countries ${ }^{5-6}$. Another important component of environment is soil which is the region on earth's crust where geology and biology meet the land surface that provides a home to plant, animal and microbial life ${ }^{7}$. Soil teems with microscopic life (bacteria, fungi, algae, protozoa and viruses as well as macroscopic life such as earthworms, nematodes, mites and insects and also the root systems of plants. The number and kinds of microorganisms present in soil depend on many environmental factors: amount and type of nutrients available, available moisture, degree of aeration, $\mathrm{pH}$, temperature etc. Soil is generally a favourable habitat for the proliferation of microorganisms with micro colonies developing around soil particles. Number of microorganisms in soil habitats normally are much higher than those in fresh water or marine habitats ${ }^{8}$.Escherichia coliis a common bacteria found in the human intestine. Under certain conditions, E. coli can become pathogenic,i.e., it gains the ability to cause disease. It almost goes without saying that $E$. coliis an important model organism and has been for more than 120 years. For a long time $E$. coli has become a common resident of the environment. Moreover, as the environmental conditions are significantly different from what exists in the human intestine, $E$. coli fails to survive long outside of the human host. For these reasons, E. coli has been used as an indicator of recent fecal contamination and represents a threat to human and environmental health ${ }^{9}$.However, for rapid detection of indicator organisms in drinking water as well as in soil, the most probable number (MPN) method, which is not that common in usage, might be considered ${ }^{10-11}$. It is actually a qualitative test rather than quantitative indicating only the presence of coliforms, not their numerical presentation.

Materials and Method

Sample collection: The soil samples were collected from different places of Bangladesh like Dhaka,Chandpur, Norsingdi, Barisal, Tangail, whereas the water samples include both tap and surface water in which tap water were collected from different lacations of Dhaka City and surface water were collected from different ponds of Dhaka,Chandpur, Tangail and Mymensingh districts. Rivers include Buriganga and Dakatia. Soil samples were collected from 85 different locationsin UV-treated plastic bags and 93 water samples were collected in UV treated plastic screw capped bottles and transported to the laboratory within one hour and immediately subjected to microbiological analysis. 
Presumptive identification of E. coliby MPN method:In Most Probable Number method a series of tubes containing selective BGLB media were inoculated with test portion of soil samples using inoculum quantities of $1,0.1$ and $0.01 \mathrm{~g}$ but in case of water samples BGLB media were inoculated with test portion of samples using inoculum quantities of $1,0.1$ and $0.01 \mathrm{ml}$ and incubated at $37^{\circ} \mathrm{C}$ for 24 hours. Each tubes containing gas with yellow color was regarded as presumptive positive for coliform. Subsequent confirmatory test with selective eosinemethylene blue (EMB) and MacConkey agar media was performed.

Confirmative identification of E. colion EMB and MacConkey agar media:A loopful of culture from positive BGLB medium from eachdilution was streaked on EMB and MacConkey agar media for confirmative identification of the samples. The plates were incubated at $37^{\circ} \mathrm{C}$ for $24 \mathrm{hrs}$. Colonies with metallic green sheen on EMB and round, small,elevated colonies with pink pigmentation on MacConkey agar were thought to be Escherichia coli and picked as positive isolates for further identification.

Biochemical identification: The laboratory biochemical tests such asKligler $3 / 4 \mathrm{~s}$ iron agar (KIA) test,indole production test, citrate utilization test,methylred test and Voges-Proskauer test were used to confirm the identification of the selected colony from EMB and MacConkey agar media. Specific biochemical reactions such as fermentative metabolism, utilization of glucose, lactose, production of gases helped to identify Escherichia coli.

\section{Results and Discussion}

In this study, 32.26\% ( $\mathrm{n}=93$ )water samples and $52.94 \%(\mathrm{n}=85)$ of soil samples were presumptive for coliforms in BGLB media, whereas $30.59 \%$ soil isolates and $26.88 \%$ water isolates were confirmed to be Escherichia coli. Tables 1 and 2 show the most probable number (MPN) of samples that were inoculated in varying amounts in BGLB media.

Among 85 soil samples 40 samples that contained at least one positive in each dilution series and among 93 water samples 31 samples that contained at least one positive in each dilution series were further confirmed for the presence of $E$. coli through identification with biochemical tests. This study showed $30.59 \%$ soil isolates and $26.88 \%$ water isolates were E.coli, which conformed to expected biochemical reactions, formed round, small, elevated colonies with pink pigmentation on MacConkey agar media and round, small metallic green sheen poigmentation on EMB agar media.

The spread of diseases through fecal contamination of water sources particularly in developing and under developed countries are a common phenomenon that has been well reported ${ }^{12-13}$. Infectious diseases are more common in developing countries like Bangladesh due to poor quality of drinking water ${ }^{14-16}$. The present experiment was conducted for the determination of the both tap and surface water quality on the basis of the presence of indicator bacteria which indicates the chance of fecal contamination as well as health associated risks. This identification procedure was done by applying the MPN method which is rather a cheap and less time consuming method in the context of developing countries. Any water source used for cleaning or drinking purpose should not contain any organism of fecal origin ${ }^{17}$. Presence of enteric coliforms especially E. coli makes the water samples unsuitable for human consumption according to the guidelines set by WHO for the evaluation of bacteriological quality of drinking water ${ }^{12}$. In this study we have found the presence of E.coli both in tap and surface water which are used for drinking, bathing swimming and for many household purposes. Likewise we have isolated a considerable percentage

Table 1.MPN index and 95\% confidence limits for various combinations of positive tubes in a 3 tube dilution series using inoculum quantities of $1,0.1$ and $0.01 \mathrm{ml}$ for water sample analysis

\begin{tabular}{lcccc}
\hline Combination of positives & $\begin{array}{c}\text { MPN index } \\
\text { per } \mathrm{g}(\mathrm{ml})\end{array}$ & $\begin{array}{c}\text { Number of samples } \\
\text { showed this result }\end{array}$ & \multicolumn{2}{c}{$95 \%$ confidence limits } \\
\cline { 3 - 5 } $0-0-0$ & $<0.3$ & 32 & Lower & Upper \\
$1-0-0$ & 0.36 & 10 & 0.017 & 0.95 \\
$2-0-0$ & 0.92 & 4 & 0.14 & 3.8 \\
$3-2-1$ & 15.3 & 4 & 3.7 & 42.4 \\
$3-1-0$ & 4.3 & 4 & 0.90 & 18.4 \\
$3-3-3$ & $>110.3$ & 30 & 42.0 & - \\
$1-1-1$ & 1.14 & 2 & 0.36 & 3.8 \\
$2-1-0$ & 1.5 & 1 & 0.37 & 4.2 \\
$3-3-0$ & 24.3 & 2 & 4.2 & 100.4 \\
$3-0-0$ & 2.3 & 1 & 0.46 & 9.4 \\
$3-3-1$ & 46.3 & 2 & 9.0 & 200.4 \\
$3-2-0$ & 19.3 & 1 & 1.8 & 42.4 \\
\hline
\end{tabular}


Table 2. MPN Index and 95\% Confidence Limits for various Combinations of positive tubes in a 3 tube dilution series using inoculum quantities of $1,0.1$ and $0.01 \mathrm{~g}$ for soil sample analysis

\begin{tabular}{lcccc}
\hline Combination of positives & $\begin{array}{c}\text { MPN index } \\
\text { per } \mathrm{g}(\mathrm{ml})\end{array}$ & $\begin{array}{c}\text { Number of samples } \\
\text { showed this result }\end{array}$ & \multicolumn{2}{c}{$95 \%$ confidence limits } \\
\cline { 3 - 5 } $3-3-2$ & 110.3 & 6 & 18.0 & Upper \\
$1-3-0$ & 1.6 & 1 & 0.45 & 410.4 \\
$1-0-1$ & 0.72 & 1 & 0.13 & 4.2 \\
$1-0-2$ & 1.1 & 1 & 0.36 & 3.8 \\
$3-1-0$ & 4.3 & 2 & 0.90 & 3.8 \\
$3-3-3$ & $>110.3$ & 45 & 42.0 & - \\
$1-1-1$ & 1.14 & 1 & 0.36 & 3.8 \\
$3-2-3$ & 29.3 & 3 & 9.0 & 200.4 \\
$3-3-1$ & 46.3 & 3 & 9.0 & 100.4 \\
$3-0-0$ & 2.3 & 1 & 0.46 & 9.4 \\
$3-3-1$ & 46.3 & 2 & 9.0 & 200.4 \\
$3-2-1$ & 15.3 & 19 & 3.7 & 42.4 \\
\hline
\end{tabular}

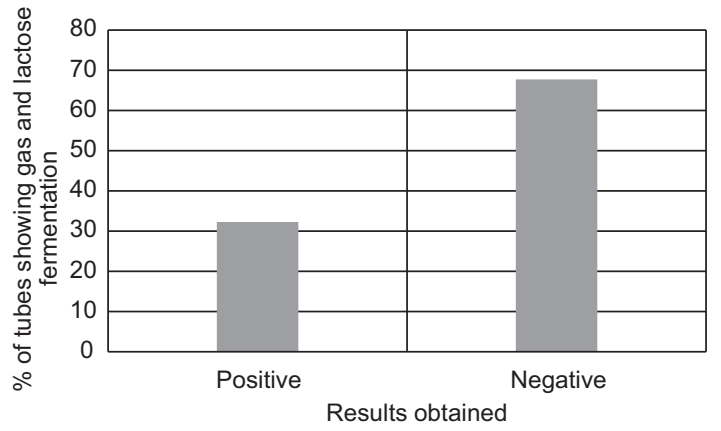

Figure 1: Result of 3 tubes dilution series of water samples using inoculum quantities of 1.0, 0.1 and $0.01 \mathrm{ml}$.

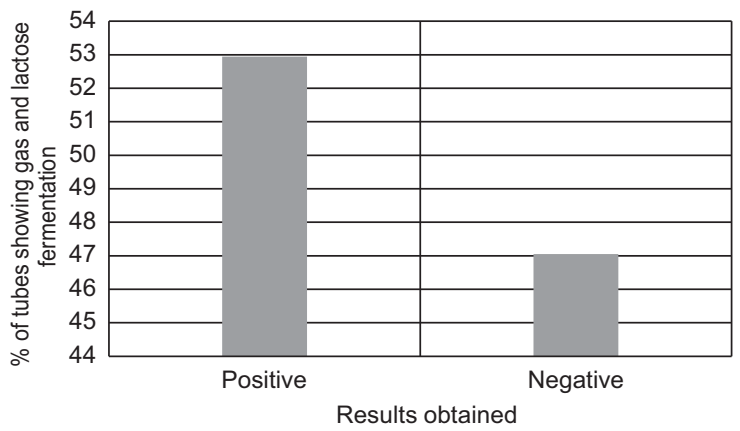

Figure 2: Result of 3 tubes dilution series of soil samples using inoculum quantities of 1.0, 0.1 and $0.01 \mathrm{~g}$.

of E.coli from soil samples collected from different places of Bangladesh.

\section{Acknowledgements}

The author would like to express thanks to the department of Microbiology,University of Dhaka for laboratory facilities.

\section{References}

1. Lamikanra A. 1999. Essential Microbiology for Students and Practitioner of Pharmacy, Medicine and Microbiology, $2^{\text {nd }}$ edn, p 406. Amkra Books, Lagos.
2. Nakade DB. 2013. Assessment of bacteriological quality of water in Kolhapur city of Maharashtra, India. Int Res J Environ Sci.2:63-65.

3. WHO. 2000. Global water supply and sanitation assessment 2000 report.

4. WHO guidelines for drinking water quality. 1993. Geneva, World Health Org.1:1-29.

5. Manja KS, Maurva MS and Ran KM. 1982. A simple field test for the detection of fecal pollution in drinking water. Bull WHO.60:797-801.

6. Geldreich EL. 1992. Water borne pathogens invasions: A case for water quality protection in distribution.Proceedings of American Water Works Association. Water Quality Technology Conference 1-18.

7. Pelczar MJ, Chan ECS and Krieg NR. 1993. Microbiology: Concept and Application International edition McGraw-Hill, USA: 281-324.

8. Atlas RM and Bartha R. 1998. Microbial Ecology: Fundamentals and Applications. $4^{\text {th }}$ Edn, p 300-350. Benjamin Cummings Publishing Company, Inc. California.

9. Hartstra AV, Bouter KEC, Backhed F and Nieuwdorp M. 2015. Insights into the role of the microbiome in obesity and type 2 diabetes. Diabetes Care.38(1):159-165.

10. Goel S, R Sood, S Mazta, P Bansal andA Gupta.2007. Bacteriological quality of water samples of a tertiary care medical center campus in north western Himalayan region of India. Inter J Third World Med. 5(1):DOI: $10.5580 / 10 \mathrm{~d} 5$.

11. Abera S,Zeyinudin A, Kebede B, Deribew A, AliS and Zemene E. 2011. Bacteriological analysis of drinking water sources. Afr J Microbial Res. 5(18):2638-2641.

12. World Health Organization. 1996. Guidelines for drinking water quality: Health criteria and other support information. p18-97.World Health Organization, Geneva.

13. OloweOA, OjurongbeO, OpaleyeOO, AdedosuOT, OloweRA andEniolaKIT. 2005. Bacteriological quality of water samples in Osobometropolis. Afr J ClinExpMicrobiol.6(3):219-222.

14. AhmedT, Baidya S, Acharjee M and Rahman T. 2013. Microbiological study of drinking water: Qualitative and quantitative approach. Asian $J$ Microbiol Biotech Env.Sc.15(4): 647-654.

15. AcharjeeM, JahanF, RahmanF and NoorR. 2013. Bacterial proliferation in municipal water supplied in Mirpur locality of Dhaka City, Bangladesh. Clean-Soil Air Water.41:1-8.

16. AcharjeeM, Rahman F, Beauty S, Feroz F, Rahman M and Noor, R. 2012. Microbiological study on supply water and treated water in Dhaka city. Stam J Microbial.1(1):42-45.

17. BondeGJ. 1977. Bacterial Indication of Water Pollution in Advances in Aquatic Microbiology. Academic Press, London. 